Xue GAO,PhD

School of Economics and Management

Email: 847786221@qq.com

Professor Yixin REN, PhD

School of Economics and Management

Shandong University of Science and Technology

Email: 912393320@qq.com

Xin LI, PhD

Antai College of Economics \& Management

Shanghai Jiao Tong University

Email: shin_li@foxmail.com (Corresponding Author)

Associate Professor Xiaoyan ZHANG, PhD

National Academy of Economic Strategy

Chinese Academy of Social Sciences

\title{
MULTIPLE SPILLOVERS OF ECONOMIC POLICY UNCERTAINTY: A TRANSNATIONAL ANALYSIS
}

\begin{abstract}
: we test the spillover effect of the economic policy uncertainty (EPU) involving 21 countries by using a general spatial autoregression model. We provide evidence that the result of the VAR model is too complex to derive common regularities even after Lasso dimensionality reduction, while the spatial model is helpful to taking into account more information for the improvement of predication. The dynamic evolution of the spillovers shows the spillover effect of geographic dependence is weakening in the long run, while the role of economic dependence becomes increasingly prominent with global economic integration. Furthermore, we find spillovers show breaks during crises and show asymmetry for higher EPU countries. Our research helps economic participants to enhance their expectations of EPU, which is of great significance in many aspects of economic activity.

Keyword: Multiple Dependence; Spatial Estimation; Dynamic Evolution; Generalized Method of Moments; Lasso Regression; Asymmetric Spillovers.
\end{abstract}

\section{JEL Classification: C21; D80; F42}

\section{Introduction}

The research on the economic policy uncertainty (EPU) and its impact has increased rapidly since the 2008 financial crisis. Especially, a large amount of literatures begins to empirically test how EPU affects economic variables since the measurement of EPU was proposed by Baker et al. (2016).Such as Kang et al. (2014) examine the impact of EPU on corporate investment behavior; Liu and Zhang (2015) examine the impact of EPU on the stock market and Brogaard and Detzel (2016)

DOI: 10.24818/18423264/54.2.20.06 
examines the asset-pricing implications of EPU. In addition, researchers apply EPU to macroeconomic and microeconomics dimensions such as the unemployment (Caggiano et al., 2017), the exchange rate (Krol, 2017) and the housing market (Antonakakis et al., 2015). However, most of the above studies tend to take policy shocks as exogenous and examine its effects on important variables of the economic system. Such as Pástor and Veronesi (2013) assume that information about policy changes is visible only to policymakers and cannot be predicted by investors when they constructing a theoretical model to describe how EPU affects stock returns. This underlying assumption leads us to consider an important question of whether the EPU is predictable if it turns out to have important impacts on the economy?

In the case of financial market, if the EPU can be expected by investors, thenthey can adjust their investment strategy according to the prior expectation to avoid the loss caused by uncertainty. In this sense, the prediction of EPU is of great significance to the decision-making of economic subjects.

However, unfortunately, we have not found systematic studies of the EPU prediction at present.We argue that this may be due to the following reasons.First, under the traditional economic paradigm, literatures pay more attention to the prediction of economic variables rather than the behavior of policymakers (such as $\mathrm{Su}$ et al., 2019a). By default, policymakers are excluded from the economic system and the inherent regularity of policymaking is ignored. Second, it may be more difficult to predict policymaking than common economic variables.The reason is that policymakers may need to consider more comprehensive factors such as politics and geography besides economic factors. For example, the economic sanctions imposed by the U.S. on China in recent years may not only stem from economic factors, but may also include more political considerations at the national strategic level. Meanwhile, policy changes are usually targeted. For example, a policy that restraining purchase may be aimed at curbing house prices, while a policy that adjusting interest rates may be aimed at curbing inflation. This makes it possible that a specific combination of predictors may be valid only for a specific period of time, and it is difficult to maintain long-term validity with the change of economic environment and policy objectives.Thirdly, there may be exogenous structural changes in EPU. For example, this uncertainty is usually higher during crises, although crises may not be inherent in the domestic economic system, but are subject to transnational exogenous shocks.

However, an intuitive logic is that under the trend of globalization, the policy changes of one country are likely to have impacts on the economic policy

DOI: $10.24818 / 18423264 / 54.2 .20 .06$ 
Multiple Spillovers of Economic Policy Uncertainty: A Transnational Analysis

formulation of other countries, that is, spillover effect. If the U.S. raises trade tariffs on China, it will directly lead China to take strong counter-measures. Among the few literatures, Klößner and Sekkel (2014) examine the EPU spillover effect among six developed countries, which provides a good reference for our study. In addition, there are also some references concerning the international transmission of uncertainty (Mumtaz and Theodoridis, 2012; Colombo, 2013).However, the above literature usually considers fewer countries or regions based on the vector autoregression (VAR) family models. Mumtaz and Theodoridis (2012) examine the interaction between US and UK macroeconomic fluctuations based on the SVAR model; Colombo (2013) also examines the interaction between US and Euro area EPU based on the SVAR model.However, in view of the universal interconnectedness of globalized countries, it is difficult to incorporate these approaches into lots of countries without model over-fitting and guaranteeing degree of freedom. At the same time, the above literature mainly measures the spillover effect of uncertainty from the economic point of view, but whether such channels as geography and politics can be ignored is not yet known.

In order to overcome these problems, we use a more general method to examine the spillover effect of EPU. Based on data availability, we examined the mutual conduction and spillover effects of EPU in 21 countries. First, similar to the common practice in literature, we use a VAR (1) model for the investigation. In order to overcome the over-fitting phenomenon of the VAR model that is often criticized by literatures, we use Lasso regression algorithm to reduce the data dimension. We further visualize EPU linkages to illustrate the complexity of this relationship. Second, based on the research of Su et al. (2019b), we validate the general structural changes in linear models by using supF, aveF and expF statistics. Third, considering the complexity of the network, we use the modified GMM method proposed by Arnold et al. (2013) to construct a multidimensional spatial autoregressive model. We examine the transnational spillover effect of EPU from three dimensions: geographical dependence, economic dependence and political dependence, and adopt rolling windows approach to overcome potential structural changes. Fourthly, based on the high-dimensional spatial autoregressive model, we further investigate the asymmetry of spillover effects from high and low EPU countries. Our results show that the spillover effect estimation based on VAR model is still complex even after dimensionality reduction, and it is difficult to measure the spillover effect when number of countries increasing. The spatial autoregressive model based on high-dimensional dependency doing better in testing the spillover

DOI: 10.24818/18423264/54.2.20.06 
effect of various dependent channels and their dynamic evolution under the rolling window approach.

The rest of this paper is organized as follows. Section 2introducesthe construction of the VAR model and the Lasso method, as well as the construction of the spatial model; Section 3discusses data and spatial weighted matrices; after which Section 4 introduces the corresponding empirical result. Section 5 concludes the paper.

\section{Methodology}

The traditional approaches for the investigation of the spillover effect of uncertainty is using the VAR family models. A VAR(1) model with $n$ countries is constructed as follows:

$E P U_{t}=\beta E P U_{t-1}+u_{t}, \quad t=1, \ldots, T$

where $E P U_{t}=\left[E P U_{1, t}, \ldots, E P U_{n, t}\right]^{\prime}$ is a $n \times 1$ vector at time $t ; u_{t}=$ $\left[u_{1, t}, \ldots, u_{n, t}\right]^{\prime}$ is a $n \times 1$ random disturbance vector and $\beta$ is a $n \times n$ coefficient matrix:

$\beta=\left[\begin{array}{ccc}\beta_{1,1} & \cdots & \beta_{1, n} \\ \vdots & \ddots & \vdots \\ \beta_{n, 1} & \cdots & \beta_{n, n}\end{array}\right]$

$\beta_{i, j}$ is the coefficient of $E P U_{j, t-1}$ for the $i$-th equation. Besides the variance of $u_{t}$ and intercepts, we have to estimate $n^{2}$ coefficientsthat may raise problems called "the curse of the dimension" when $n$ is large. With the increase of dimension, the number of samples needed for analysis will increase exponentially. Moreover, in high-dimensional data space, prediction will become no longer easy, and may lead to model over-fitting.In this case, the Lasso regression is an appropriate data dimension reduction method. Lasso is based on penalty method to select variables from sample data. By compressing the original coefficients, the original small coefficients are directly compressed to 0 , thus the variables corresponding to these coefficients are regarded as non-significant variables, which are discarded directly. Rapach et al. (2013) use Lasso for the estimation of the impact of the U.S. financial market on international stock market. The Lasso method introduces penalties when estimating Equation (1):

$$
\hat{\beta}=\arg \min _{\beta \in R^{d}}|| E P U_{t}-\beta E P U_{t-1}||^{2}+P_{\lambda}(|\beta|)
$$

where penalties $P_{\lambda}(|\beta|)=\lambda \sum_{j=1}^{d}\left|\beta_{, j}\right|^{m}$ for $m \geq 0 ; \lambda$ is an adjustment parameter 
Multiple Spillovers of Economic Policy Uncertainty: A Transnational Analysis

(or vector). When $m=1$, we call $P_{\lambda}(|\beta|)$ as the Lasso penalty. Therefore, we get the lasso estimator:

$$
\hat{\beta}_{\text {lasso }}=\min _{\beta \in R^{d}}|| E P U_{t}-\left.\beta E P U_{t-1}\right|^{2} \text { s.t. } \sum_{j=1}^{d}\left|\beta_{, j}\right| \leq \Lambda, \Lambda \geq 0
$$

which is equivalent to

$$
\hat{\beta}_{\text {lasso }}=\min _{\beta \in R^{d}}|| E P U_{t}-\left.\beta E P U_{t-1}\right|^{2}+\lambda \sum_{j=1}^{d}|\beta, j|
$$

where $\Lambda$ corresponds to $\lambda$ one by one. Suppose $\Lambda_{0}=\sum_{j=1}^{d}\left|\hat{\beta}_{, j}(O L S)\right|$, when $\Lambda<$ $\Lambda_{0}$, some of coefficients would be compressed to 0 .

The estimated coefficient matrix $\hat{\beta}$ builds up a network since it measures how EPU of different countries affect each other. Using the Lasso method, the network is simplified by eliminating edges that are not different from zero. However, the network may still be too complex to get regular predictions. A more general approach is to incorporate potential linkages into the model as weight matrices based on spatial econometrics. Following Arnold et al. (2013), we construct a general multiple weighted matrices spatial auto regression as follows:

$E P U_{t}=\rho_{1} W_{1} E P U_{t}+\cdots+\rho_{k} W_{k} E P U_{t}+u_{t}, \quad t=1, \ldots, T$

where $W_{1}, \ldots, W_{k}$ are $k$ spatial weighted $n \times n$ matrices; $\rho_{1}, \ldots, \rho_{k}$ are un-estimated dependence parameters that measure spillover effects of $W_{1}, \ldots, W_{k}$. Based on Equation (3), the variance-covariance matrix $(V)$ of $E P U_{t}$ is:

$$
V=\left(I_{n}-\rho_{1} W_{1}-\cdots-\rho_{k} W_{k}\right)^{-1} \Sigma\left(I_{n}-\rho_{1} W_{1}^{\prime}-\cdots-\rho_{k} W_{k}^{\prime}\right)^{-1}(4)
$$

where $\Sigma$ is defined as the variance-covariance matrix of $u_{t}: \operatorname{Cov}\left(u_{t}\right)=$ $\operatorname{diag}\left\{\sigma_{1}^{2}, \ldots, \sigma_{n}^{2}\right\}$, the matrix $I_{n}-\rho_{c} W_{c}-\rho_{m} W_{m}-\rho_{b} W_{b}-\rho_{g} W_{g}$ is reversible under some reasonable assumptions (Arnold et al., 2013). The model in Equation (3) contains $n+k$ parameters: the $k$ correlation parameters $\rho_{1}, \ldots, \rho_{k}$ and $n$ parameters of variance, $\sigma_{i}^{2}$.

The estimation of this model using the maximum likelihood estimation is computationally expensive and it may inconsistent if the heteroscedasticity is not taken into account (Lin and Lee, 2010). In this paper, we follow Arnold et al. (2013) and implement a two-step estimation procedure to simplify calculation. In the first step, we estimate $\rho_{c}, \rho_{m}, \rho_{b}$ and $\rho_{g}$ using generalized method of moments (GMM) proposed by Kelejian and Prucha (1999) and Kapoor et al. (2007). Arnold et al. (2013) show that the first step does not depend on the parameters of variance.

DOI: 10.24818/18423264/54.2.20.06 
Xue Gao, Yixin Ren, Xin Li, Xiaoyan Zhang

In the second step, we estimate the variance parameters through the estimated value of $\rho_{c}, \rho_{m}, \rho_{b}$ and $\rho_{g}$. For the first step, the moment conditions of the GMM estimator is:

$\mathrm{E}\left(u_{t}^{\prime} W_{1} u_{t}\right)=\operatorname{tr}\left(W_{1} \Sigma\right)=0$
$\vdots$
$\mathrm{E}\left(u_{t}^{\prime} W_{k} u_{t}\right)=\operatorname{tr}\left(W_{k} \Sigma\right)=0$

Based on Arnold et al. (2013), we have

$\Gamma \lambda+\gamma=0$

where $\lambda$ is a $\left(k^{2}+3 k\right) / 2 \times 1$ vector:

$\lambda=\left[\rho_{1}, \ldots, \rho_{k}, \rho_{1}^{2}, \ldots, \rho_{1}^{2}, \rho_{1} \rho_{2}, \ldots, \rho_{1} \rho_{k}, \rho_{2} \rho_{3}, \ldots, \rho_{2} \rho_{k}, \ldots, \rho_{k-1} \rho_{k}\right]^{\prime}$

and $\Gamma$ is a $k \times\left(k^{2}+3 k\right) / 2$ matrix. For $i, j \in\{1, \ldots, k\}$,

$$
\begin{gathered}
\Gamma_{i, j}=\mathrm{E}\left(-r_{t}^{\prime}\left(W_{i}+W_{i}^{\prime}\right) W_{j} r_{t}\right) \\
\Gamma_{i, k+j}=\mathrm{E}\left(r_{t}^{\prime} W_{j}^{\prime} W_{i} W_{j} r_{t}\right)
\end{gathered}
$$

In addition, for $l \in\left\{1, \ldots,\left(k^{2}+3 k\right) / 2\right\}$, the representation of $\Gamma_{i, l+2 k}$ is corresponding to the $(l+2 k)$-th element of $\lambda$. For example, corresponding to $\rho_{p} \rho_{q}(p, q \in\{1, \ldots, k\}), \Gamma_{i, l+2 k}$ is represented as:

$$
\Gamma_{i, l+2 k}=\mathrm{E}\left(-r_{t}^{\prime} W_{p}\left(W_{i}+W_{i}^{\prime}\right) W_{q} r_{t}\right)
$$

The expectation operator in the matrix $\Gamma$ and the vector $\gamma$ can be replaced by the sample average ( $\hat{\Gamma}$ and $\hat{\gamma}$ ), then we have the GMM estimator of $\rho$ as:

$\hat{\rho}_{G M M}=\left(\hat{\rho}_{1}, \ldots, \hat{\rho}_{k}\right)_{G M M}^{\prime}=\arg \min _{\rho \in S}|| \hat{\Gamma} \lambda+\hat{\gamma} \| \mid$

Arnold et al. (2013) prove that the GMM estimator shows consistency and asymptotic normality for $T \rightarrow \infty$,

$$
\begin{gathered}
\hat{\rho}_{G M M} \stackrel{p}{\rightarrow} \rho_{0}, \quad \rho_{0}=\left[\rho_{1}, \ldots, \rho_{\mathrm{k}}\right] \\
\sqrt{T}\left(\hat{\rho}_{G M M}-\rho_{0}\right) \stackrel{d}{\rightarrow} N\left(0, d_{0}^{-1} S_{W}\left(d_{0}^{-1}\right)^{\prime}\right)
\end{gathered}
$$

where

$$
\begin{gathered}
d_{0}=\mathrm{E}\left(\frac{\partial(\hat{\Gamma} \lambda+\hat{\gamma})}{\partial \rho}\left(r_{1}, \rho_{0}\right)\right)=\Gamma \lambda^{(1)} \\
S_{W}=\sum_{t=-\infty}^{\infty} \mathrm{E}\left[\left(\begin{array}{c}
u_{1}^{\prime} W_{1} u_{1} \\
\vdots \\
u_{1}^{\prime} W_{k} u_{1}
\end{array}\right)\left(\begin{array}{c}
u_{t}^{\prime} W_{1} u_{t} \\
\vdots \\
u_{t}^{\prime} W_{k} u_{t}
\end{array}\right)\right]
\end{gathered}
$$


Multiple Spillovers of Economic Policy Uncertainty: A Transnational Analysis

and $\lambda^{(1)}$ is a $\left(k^{2}+3 k\right) / 2 \times k$ matrix. For $i, j \in\{1, \ldots, k\}$,

$$
\begin{gathered}
\lambda_{i, i}^{(1)}=1, \\
\lambda_{i+k, i}^{(1)}=2 \rho_{i} \\
\lambda_{i+k, j}^{(1)}=0, i \neq j
\end{gathered}
$$

In addition, for $l \in\left\{1, \ldots,\left(k^{2}+3 k\right) / 2\right\}$, the representation of $\Gamma_{i, l+2 k}$ is corresponding to the $l+2 k$-th element of $\lambda$. For example, corresponding to $\rho_{p} \rho_{q}$ $(p, q \in\{1, \ldots, k\})$,

$$
\lambda_{l+2 k, i}^{(1)}=\left\{\begin{array}{l}
\rho_{q}, \text { if } i=p \\
\rho_{p}, \text { if } i=q \\
0, \text { otherwise }
\end{array}\right.
$$

Based on the above analysis, we can estimate $\hat{\rho}_{G M M}$ and make its statistical inference to test the significance of $\hat{\rho}_{G M M}$.Furthermore, we can use the rolling window method for the investigation of the time-varying spillover effect. It should note that other approaches such as the recursive based methods (Su et al., 2017)are also useful for the investigation, but the later one generally need more compute and it is time-consuming.

\section{Data and Spatial Weighted Matrices}

We use the data of news based EPU from Baker et al. (2016), who build up a database that is readily available at the policy uncertainty website. ${ }^{1}$ Our sample is monthly for the period of March2003 to December 2018 and comprises 21 countries. See Baker et al. (2016) for more details about the measurement of the EPU indices. Figure 1 plots the dynamic movement of EPU for the 21 countries. We use hierarchical clustering to bring together countries with similar EPU trends as far as possible. See Murtagh and Legendre (2014) for more details about the hierarchical cluster. It can be seen that EPUs in different countries have very similar long-term trends in the sample period, which provides us a very strong intuition of transnational spillover effects. Figure 2 also plots that heat map of the correlation matrix of EPUs, which is sorted by the result of the hierarchical cluster. The density curve in the color bar shows that the correlation coefficients are mostly positive, with an average of about 0.5.The short-term fluctuations of EPU are also very similar in many countries (such as the U.S. and Korea), which further provides

\footnotetext{
${ }^{1} \mathrm{http}: / / \mathrm{www} \cdot$ policyuncertainty.com.
} 
Xue Gao, Yixin Ren, Xin Li, Xiaoyan Zhang

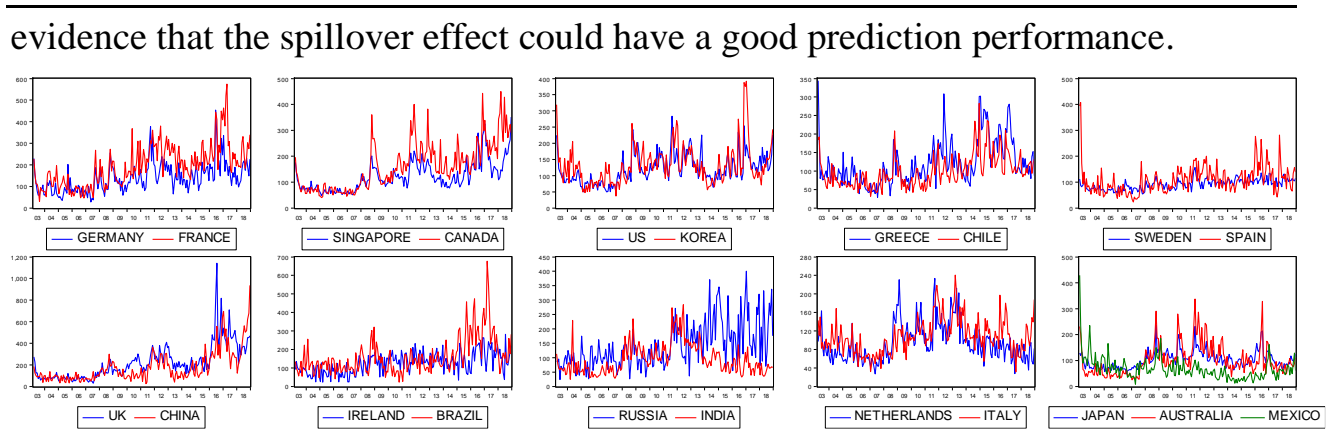

Figure 1. EPU of 21 Countries

Heat Map of the Correlation Matrix with Hierarchical Clustering

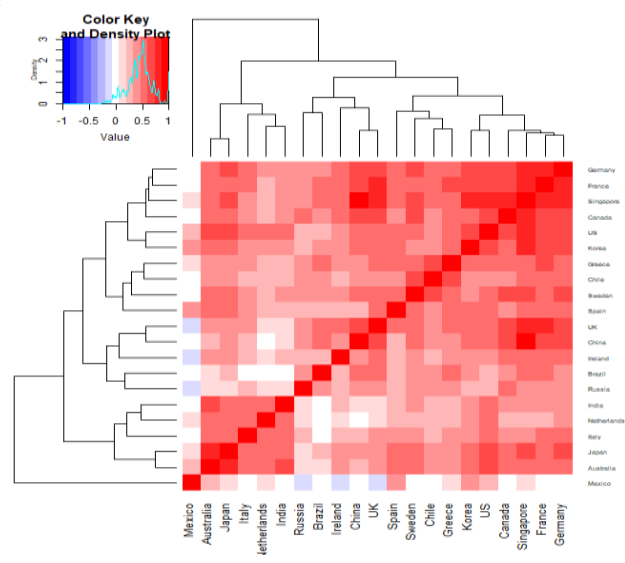

Figure 2. Heat Map of the Correlation Matrix with Hierarchical Clustering

When the number of countries involved is large, it is difficult to incorporate all EPU information into the VAR model while ensuring the simplicity of the model. By contrast, estimating spillover effects by introducing spatial weighting matrices can not only simplify the estimation of model parameters to a great extent, but also incorporate more information besides EPU by setting different spatial weighting matrices. More importantly, multi-dimensional spatial regression estimation can help to compare the spatial spillover effects of different channels within a unified model framework. In this paper, we mainly consider three dimensions of uncertainty spillover channels: geographical dependence, macroeconomic dependence and political dependence. ${ }^{2}$ We construct these spatial matrices as follows.

\footnotetext{
${ }^{2}$ In fact, we also considered the financial market dependence, while its spillover effect is pretty low that shows no difference with zero. This may be because the overall economic interdependence covers financial channels to a large extent.
} 
Multiple Spillovers of Economic Policy Uncertainty: A Transnational Analysis

(1) Geographical weighted matrix. Referring to most literatures, the reciprocal of the geographical distance between the two countries is taken as an element of the geographic weight matrix. Previous studies mostly used the distance between capitals as geographical distance. We use the longitude and latitude information of capitals to calculate the spherical distance between the two capitals. Specifically, we use the following Haversine formula for the calculation:

$$
\text { haversine }\left(\frac{d}{R}\right)=\text { haversine }\left(\left|\Phi_{1}-\Phi_{2}\right|\right)+\cos \left(\phi_{1}\right) \cos \left(\phi_{2}\right) \text { haversine }\left(\left|\phi_{1}-\phi_{2}\right|\right)(9)
$$

where $\left(\Phi_{1}, \phi_{1}\right)$ is the longitude and latitude of capital $\mathrm{A}$ and $\left(\Phi_{2}, \phi_{2}\right)$ is the longitude and latitude of capital $\mathrm{B} ; d$ is the spherical distance between $\mathrm{A}$ and $\mathrm{B} ; R$ is the radius of the earth.

(2) Macroeconomic weighted matrix. The macroeconomic weight is measured by the reciprocal of the macroeconomic distance. Traditionally, there are two ways to measure macroeconomic distance: based on GDP per capita and based on GDP. In this paper, we argue that the spillover effect of EPU should be greatly influenced by a country's total economic output. For example, as a developing country, China's per capita GDP ranks relatively low, but its total economic output is second only to that of the U.S. and it is obvious that the impact of China's economic policy changes on the world cannot be ignored. The macroeconomic distance of country A and B $\left(E D_{A B}\right)$ is calculated as follows:

$E D_{A B}=\frac{\left|G D P_{A}-G D P_{B}\right|}{\left(G D P_{A}-G D P_{B}\right) / 2}$

where $G D P_{A}$ and $G D P_{B}$ are the average GDP during the sample period. The data of GDP sources from the world bank database.

(3) Political weighted matrix. The political weight is measured by the reciprocal of the political distance. The measure of the political distance in this paper refers to Berry et al. (2010), we calculate the political distance between the two countries from three dimensions: the political stability, the degree of democracy and the size government of the host country. Among them, the degree of democracy is measured by the number of independent bodies with veto power (data sources from the POLCONV database); the degree of democracy is measured by the national democracy score (data sources from the Freedom House database); the size government is measured by the share of government consumption expenditure in GDP. The data of government consumption expenditure sources from the world bank database. Following Berry et al. (2010), we use the Mahalanobis distance method to aggregate these indicators as the political distance. 


\section{Empirical Results}

Follows literatures introduced before, we first use the VAR(1) model from Equation (1) to estimate how EPUs in 21 countries affect each other. As mentioned earlier, we use the Lasso method for the data dimension reduction. This is necessary because for a VAR(1) model with intercepts that including 21 countries, we have to estimate 462 parameters using the limited monthly data during the period of March 2003 and December 2018 (190 months). The estimation of the VAR(1) model using the Lasso method is based on the Lasso penalty. We combine and estimate all possible regression factors, and select the estimated value of beta when the penalty likelihood function is minimum as the final parameter estimate.In this paper, we set the adjustment parameter $\lambda$ (mentioned in Section 2) as 0.01. The purpose of the VAR(1) regression is to show that it is difficult to cope with the large number of countries, even after using Lasso method for data dimensionality reduction. We present the estimation result visually through converting the coefficient matrix $\hat{\beta}_{\text {lasso }}$ into a network graph. Figure 3 shows the result, which is based on the force-directed layout algorithm proposed by Fruchterman and Reingold (1991).

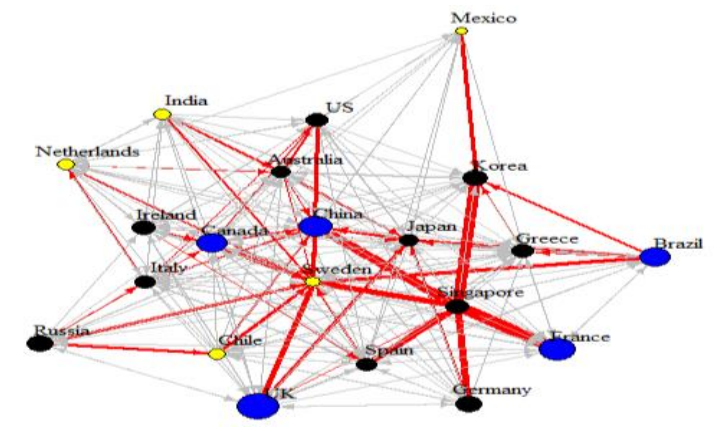

Figure 3. Network Visualization of the VAR(1) System using Lasso Regression In Figure 3, the size of vertices is weighted by the average value of EPU for each country during the sample periods. In addition, the size of edges is weighted by the value of estimated coefficients. Furthermore, in order to make coefficients comparable, we row standardized the coefficient matrix $\hat{\beta}_{\text {lasso }}$. It can be seen that even after Lasso dimensionality reduction, the estimation results of VAR model are still very complex.This fully proves that the VAR model is hard to present clear result when there are more countries involved. Furthermore, the VAR model we estimated above does not introduce more information through controlling more variables. Intuitively, this will also limit the predictive power of the model. For example, an intuitive idea is that countries with higher policy uncertainty may have 
Multiple Spillovers of Economic Policy Uncertainty: A Transnational Analysis

more spillover effects when other conditions remain unchanged. This could lead to an asymmetry of EPU's spillover effects. We incorporate this idea into Figure 3 by highlighting higher standardized coefficients (edges) into red color. Specifically, all standardized coefficients (edges) that is higher than the $75 \%$ quantile is highlighted as blue, and all coefficients that is lower than the $25 \%$ quantile is highlighted as yellow. Some potential regularities may be revealed through the connection of vertex and edge size.In general, it seems that vertices with larger size (blue, higher average EPU)relate to thicker edges (higher standardized coefficients), while vertices with lower size (yellow, lower average EPU)are generally marginalized, thus reflecting some kinds of asymmetry. We will further discuss it as an expansive research in the last part of the empirical analysis. An exception is Singapore, whose average EPU is about in the middle level but seems to be related to the most spillover effects.Some possible explanations are as follows. First, Singapore has implemented a managed floating exchange rate system since 1980, which limits its monetary policy autonomy to a certain extent and increases its dependence on external policy changes. Second, the economic growth of Singapore mainly relies on shipping, transit trade and tourism, which makes its policy-making more vulnerable to changes in the external environment.

An underlying assumption cannot be ignored in the full-sample test; that is, all parameters are not varying over time (constant). However, in practical cases, this underlying assumption cannot be satisfied because the universal existence of structural changes will make the results of the full-sample test unreliable. To test the parameter stability, we use the supF, aveFandexpF tests. Among them, the supF test is under the null hypothesis of parameter constancy against a one-time sharp shift in parameters; the aveF and expF tests are carried out under the null hypothesis that parameters follow a Martingale process against the possibility that the parameters might evolve gradually. In addition, the fraction of the sample in $(0.15,0.85)$ is used to ensure fulfillment of the $15 \%$ trimming requirement of these tests. For each equation of the VAR model, we calculate statistics and report results in Table 1. It is seen that the supF test and the expF test reject the null hypothesis of no structural changes for all countries at the 5\% significant level, and for 19 countries (except Australia and Ireland), the aveF test also suggests structural changes during the full-sample period. This provides us the evidence for the applying of the rolling window approach to test the dynamic evolution of the spillover effect. Next we will investigate the spatial dependence using a multiple weighted matrices model under the rolling window framework. 
Xue Gao, Yixin Ren, Xin Li, Xiaoyan Zhang

Table 1.Structural Changes Tests based on the VAR(1) System

\begin{tabular}{|c|c|c|c|c|c|c|c|}
\hline Country & supF & aveF & $\operatorname{expF}$ & Country & supF & aveF & $\operatorname{expF}$ \\
\hline \multirow[t]{2}{*}{ Australia } & $48.459 * * *$ & 25.404 & $20.496 * *$ & India & $81.888^{* * * *}$ & $58.995 * * *$ & $36.830 * * *$ \\
\hline & $(0.008)$ & $(0.185)$ & $(0.010)$ & & $(0.000)$ & $(0.000)$ & $(0.000)$ \\
\hline \multirow[t]{2}{*}{ Brazil } & $129.404 * * *$ & $76.314 * * *$ & $60.563 * * *$ & Ireland & 34.917 & 27.596 & 15.036 \\
\hline & $(0.000)$ & $(0.000)$ & $(0.000)$ & & $(0.201)$ & $(0.104)$ & $(0.166)$ \\
\hline \multirow[t]{2}{*}{ Canada } & $116.203^{* * * *}$ & $66.090 * * *$ & $54.429 * * *$ & Japan & $54.195 * * *$ & $33.420 * *$ & $23.069 * * *$ \\
\hline & $(0.000)$ & $(0.000)$ & $(0.000)$ & & $(0.001)$ & $(0.017)$ & $(0.002)$ \\
\hline \multirow[t]{2}{*}{ Chile } & $78.345^{* * *}$ & $44.505^{* * * *}$ & 36.010 *** & Korea & $84.447 * * *$ & $40.883 * * *$ & $38.043 * * *$ \\
\hline & $(0.000)$ & $(0.000)$ & $(0.000)$ & & $(0.000)$ & $(0.001)$ & $(0.000)$ \\
\hline \multirow[t]{2}{*}{ China } & $128.036^{* * * *}$ & $51.697 * * *$ & $60.076 * * *$ & Mexico & $68.459 * * *$ & $43.043 * * *$ & $31.376 * * *$ \\
\hline & $(0.000)$ & $(0.000)$ & $(0.000)$ & & $(0.000)$ & $(0.000)$ & $(0.000)$ \\
\hline \multirow[t]{2}{*}{ Germany } & $94.564 * * *$ & $32.615 * *$ & $43.077 * * *$ & Netherlands & $42.810 * *$ & $33.290 * *$ & $18.867 * *$ \\
\hline & $(0.000)$ & $(0.022)$ & $(0.000)$ & & $(0.036)$ & $(0.017)$ & $(0.026)$ \\
\hline \multirow[t]{2}{*}{ Italy } & $55.875^{* * *}$ & $38.795 * * *$ & $24.094 * * *$ & Russia & $45.163^{* *}$ & $34.099 * *$ & $19.846^{* * *}$ \\
\hline & $(0.001)$ & $(0.002)$ & $(0.001)$ & & $(0.020)$ & $(0.013)$ & $(0.015)$ \\
\hline \multirow[t]{2}{*}{ UK } & $170.629 * * *$ & $70.063 * * *$ & $81.110 * * *$ & Singapore & $119.192 * * *$ & $52.273 * * *$ & $55.889 * * *$ \\
\hline & $(0.000)$ & $(0.000)$ & $(0.000)$ & & $(0.000)$ & $(0.000)$ & $(0.000)$ \\
\hline \multirow[t]{2}{*}{ France } & $85.252 * * *$ & $34.146 * *$ & $38.422 * * *$ & Sweden & $58.780 * * *$ & $33.656 * *$ & $25.427 * * *$ \\
\hline & $(0.000)$ & $(0.013)$ & $(0.000)$ & & $(0.000)$ & $(0.015)$ & $(0.000)$ \\
\hline \multirow[t]{2}{*}{ Spain } & $71.973 * * *$ & $53.517 * * *$ & $32.754 * * *$ & US & $44.018 * *$ & $31.511 * *$ & $19.091 * *$ \\
\hline & $(0.000)$ & $(0.000)$ & $(0.000)$ & & $(0.026)$ & $(0.032)$ & $(0.023)$ \\
\hline \multirow[t]{2}{*}{ Greece } & $87.392 * * *$ & $43.756^{* * * *}$ & 39.823 *** & & & & \\
\hline & $(0.000)$ & $(0.000)$ & $(0.000)$ & & & & \\
\hline
\end{tabular}

Note: $* * *$ and $* *$ denotes significance at the $1 \%$ and $5 \%$ level, respectively; $\mathrm{p}$-values are in parentheses.

As discussed before, we use the model (Equation (3)) proposed in Section 2 for the spatial econometric investigation. The two-step GMM based estimation is utilized for the investigation to simplify the calculation. In addition, when calculating $S_{W}$ with expected operators, we use a kernel-based variance estimator proposed by de Jong and Davidson (2000). Specifically, we use the Bartlett kernel and bandwidth of $\log (T)$ for the estimation.Using the 3 weighted matrices discussed in Section 3, we build up a spatial model with 3 kinds of dependences. Though does not repot as a table, we estimate the full-sample data and the result shows that spatial coefficients 
Multiple Spillovers of Economic Policy Uncertainty: A Transnational Analysis

are $0.277,0.551$ and 0.328 for the geographical dependence, macroeconomic dependence and political dependence, respectively. The $t$ statistics of 3.773, 6.644 and 13.986 suggest the estimation shows significance at the $1 \%$ level. It can be seen that the estimation based on spatial weight matrix greatly reduces the loss of model freedom compared with VAR model. In addition, from the estimated results, economic dependence seems to bear the highest spillover effect, political dependence contributes considerably to the spillover of EPU, while geographical dependence is the weakest. This seems reasonable since in the context of global economic integration, geographic spatial constraints do not seem to be the main obstacle to economic linkages. We find that political dependence also contributes to the spillover effect of EPU, which can hardly be reflected by VAR model. Intuitionally, this phenomenon is easily recognized, and economic contests at the national level are often manifested through political means. For example, the U.S. uses administrative means to suppress Chinese high-tech companies, such as Huawei, it is more likely to be political means rather than economic policies.

Next, we use rolling windows to examine the dynamic evolution of spillover effects. Specifically, we set a fixed window with the size of 24 months ( 2 years). There is no strict standard for the setting of the window size. Generally, if the window size is too small, it is difficult to derive an accuracy estimation due to the small sample size. On the other hand, if the window width is too large, there will be a long period of sacrifice in the dynamic evolution of spillover effect. Pesaran and Timmerman (2005) argued that the optimal window size should depend on the size of structural changes. Based on the common macroeconomic time series, they suggest that the window size should be set at more than 20 . Although not presented in this paper, we also examined the window size of 36 and 48 months, the overall results do not show significant differences compared with 24 months, which confirmed the robustness if results in this paper.

Figure 4 shows the corresponding result. It should note that the estimation result sacrifices 23 months since we implement a window size of 24 . As a result, the estimated spillover effect begins from February 2005. For each time point of Figure 4 (such as April 2005), it reflects the spillover effect during the period of May 2003 and April 2005.Although policy uncertainty looks like random fluctuations, the dynamic evolution of spillover effects shows a clear long-term trend.Different from the full-sample regression, the rolling window result shows that the geographic dependence is actually shows a downward trend. In contrast, the economic dependence generally shows an upward trend.We argue that this trend can be 
explained in the overall macro context of the gradual integration of the global economy since the past decade.In the 21st century, economic development has become the primary goal of most countries, which makes the spillover effect of policy changes increasingly transmitted through the channel of economic dependence. Meanwhile, with the accelerated development of Internet technology, the impact of real geographical distance seems to be weaker. It can be seen that geographic dependence is not always lower than economic dependence as the full sample results show. Before 2012, geographic dependency has been dominant among the three dependencies, which indicates that the transnational spillover effect of EPU has a gradual regime switch process in the long run.

The political dependence shows higher volatility in the high frequency range before 2012, and its spillover effect is relatively low during this period, which suggests a limited role of the political dependence. However, the spillover effect caused by political dependence increases significantly after 2014.This may be related to the growing uncertainty of world politics in recent years such as the U.S. presidential election, Britain's departure from Europe, the failure of Italian referendum and the trade dispute between the U.S. and China. All these events could have an impact on the economic system, such as foreign exchange and trade, leading to national-level economic policy responses and strengthen the spillover effect of political dependence channels.

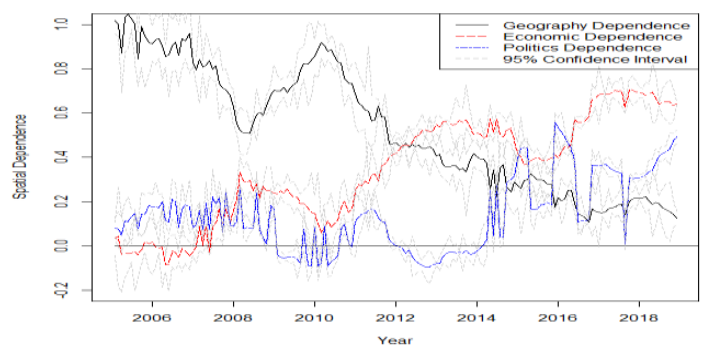

Figure 4. Dynamic Evolution of Spillovers Using the Spatial Regression

Based on the above analysis, we find that compared with VAR model, high-dimensional spatial regression based on multi-spatial dependence cannot only reduce the consumption of degrees of freedom and prevent over-fitting, but also incorporate more comprehensive information based on economic, geographical and political spatial weight matrix to help discover potential regularities and dynamic trends in transnational spillover effects of EPU.As an expanding research, we next 
Multiple Spillovers of Economic Policy Uncertainty: A Transnational Analysis

further examine how the partitioning of spatial weighting matrices can help us discover the deeper regularity of EPU spillover effect.

Another noteworthy phenomenon is that although the spillover effect of geographical dependence has maintained a downward trend in the long run, it rose rapidly during the 2008 financial crisis. Meanwhile, the spillover effect of economic dependence declined rapidly during this period. Compared with the long-term trend, this short-term structural change highlights the importance of geographical dependence. We believe that although technological advances have made geographic distance less influential in many ways, geographic dependence still needs to be taken seriously when crises occur. For example, the international trade in commodities may be more convenient between neighboring countries; during the crisis, people may prefer to travel to neighbouring countries; the national macroeconomic strategy may still largely consider geographical distance (such as the strategy of China's one belt).

In the above analysis of the network visualization of the VAR(1) system (Figure 3), we find that countries with higher average EPU during the sample period seem to relate to higher spillover effects, while countries with lower average EPU are marginalized since they mostly relate to lower spillover effects. This kind of asymmetry can be statistically test using the spatial autoregression model on the basis on the above analysis. In this paper, we pay our attention on the economic dependence since it is obviously the most important channel of spillover effect at present.For the economic weighted matrix, namely, $W_{e}$, the $i$-th row of $W_{e}$ measures the overall spillover effect of other countries to country $i$. In this case, we split two matrices from $W_{e}: W_{e}^{h}$ and $W_{e}^{l}$. We set the $i$-th column of $W_{e}^{h}$ as zero if the average EPU of country $i$ lower than $60 \%$ quantile of all countries' average EPU, otherwise it is equal to the $i$-th column of $W_{e}$. The same way, we set the $i$-th column of $W_{e}^{l}$ as zero if the average EPU of country ihigher than $40 \%$ quantile of all countries' average EPU, otherwise it is equal to the $i$-th column of $W_{e}$. By including $W_{e}^{h}$ and $W_{e}^{l}$ into the spatial autoregression model, the corresponding coefficients $\rho_{e}^{h}$ and $\rho_{e}^{l}$ measures spillover effects from high and low EPU countries, respectively.

Following the above setting, we still use a window size of 24 months to investigate the dynamic evolution of this asymmetry. Figure 5 shows the result. It is clearly seen that countries with higher EPUs exist more spillover effects. This regularity is basically stable in the whole sample period. In addition, we find that the spillover effect of countries with lower average EPU is sometimes negative (before 2012),

DOI: 10.24818/18423264/54.2.20.06 
which means that countries with lower frequency of policy changes are less likely to produce negative externalities, which could help other countries reduce policy uncertainty through economic dependences. For example, countries with stable economic policies may have lower risks in foreign exchange and international trades, which helps to form stable economic cooperation. At last, the long-term trend of Figure 5 shows similarity with Figure 4, which proves the robustness of our result.

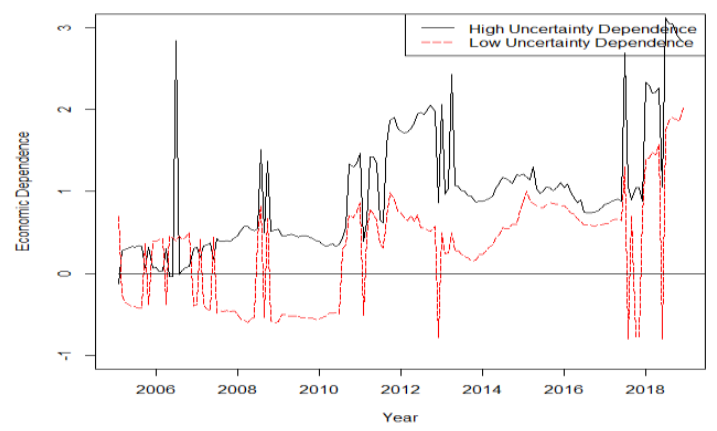

Figure 5. Asymmetry Spillovers from High and Low EPU Countries

\section{Conclusions}

This paper provides a framework for the measurement of the transnational economic policy uncertainty spillover effect in a multiple spatial dependence autoregression model. By taking into account the geographical dependence, macroeconomic dependence and political dependence, we provide evidence that the spatial based model performs better than the widely used VAR model in increasing freedom and preventing overfitting when the number of variables (or countries) is large.We believe that in the context of global economic integration, researches conducted by a small number of countries cannot give a more general and concise conclusion from the overall perspective of the economic system composed of multiple dependencies. We provide evidence that when the number of countries is large, the result of the VAR model gives a pretty complex network that cannot directly and rigorously show deeper regularities.

The main contribution of this paper is that we provide feasibility for predicting the uncertainty of economic policy.A large number of literatures on uncertainty of economic policy are mostly aimed at examining its impact on other important economic variables, potentially regarding uncertainty itself as exogenous shocks. In our analysis we test the dynamic evolution of spillover effects from different dependences. We find that the spillover effect from the geographical dependence is 
Multiple Spillovers of Economic Policy Uncertainty: A Transnational Analysis

decreasing while the channel of the economic dependence becomes increasingly important. The transnational conduction of the economic policy uncertainty is not only connected with the economy, it also relates to non-economic factors. Especially when the global economy encounters major structural shocks (the 2008 global financial crisis), the uncertainty spillover effect of geographical dependence needs to be paid enough attention. In addition, we provide evidence of the asymmetry spillover effect from high and low economic policy uncertainty countries, which provides useful information for the predication.

ACKNOWLEDGEMENTS

This research is supported by the China Postdoctoral Science Foundation (Grant No: 2019M650946).

\section{REFERENCES}

[1] Antonakakis, N., Gupta, R.\&André, C. (2015),Dynamic Co-Movements between Economic Policy Uncertainty and Housing Market Returns. Journal of Real Estate Portfolio Management, 21(1), 53-60;

[2] Arnold, M., Stahlberg, S.\&Wied, D. (2013),Modeling Different Kinds of Spatial Dependence in Stock Returns. Empirical Economics, 44(2), 761-774;

[3] Baker, S. R., Bloom, N.\&Davis, S. J. (2016),Measuring Economic Policy Uncertainty. The quarterly journal of economics, 131(4), 1593-1636;

[4] Berry, H., Guillén, M. F.\&Zhou, N. (2010),An Institutional Approach to Cross-National Distance. Journal of International Business Studies, 41(9), 1460-1480;

[5] Brogaard, J.\&Detzel, A. (2015),The Asset-Pricing Implications of Government Economic Policy Uncertainty. Management Science, 61(1), 3-18;

[6] Caggiano, G., Castelnuovo, E.\&Figueres, J. M. (2017),Economic Policy Uncertainty and Unemployment in the United States: A Nonlinear Approach.Economics Letters, 151, 31-34;

[7] Su,C. W., Khan, K., Tao, R.\& Moldovan N.C. (2019a),Does Geopolitical Risk Strengthen or Depress Oil Prices and Financial Liquidity? Evidence from Saudi Arabia. Energy, 187, 116003;

[8] Su, C. W., Wang, X. Q., Tao, R.\& LobontO.R. (2019b), Do Oil Prices Drive Agricultural Commodity Prices? Further Evidence in a Global Bio-Energy Context.Energy, 172, 691-701;

[9] Su C. W., Li., Z. Z., Chang H. L. \& LobontO.R. (2017), When Will Occur the Crude Oil Bubbles?. Energy Policy, 102, 1-12; 
Xue Gao, Yixin Ren, Xin Li, Xiaoyan Zhang

[10] Colombo, V. (2013),Economic Policy Uncertainty in the US: Does it Matter for the Euro Area?. Economics Letters, 121(1), 39-42;

[11] De Jong, R. M., \&Davidson, J. (2000),Consistency of Kernel Estimators of Heteroscedastic and Autocorrelated Covariance Matrices. Econometrica, 68(2), 407-423;

[12] Fruchterman, T. M., \& Reingold, E. M. (1991),Graph Drawing by Force-directed Placement. Software: Practice and experience, 21(11), 1129-1164;

[13] Kang, W., Lee, K.\&Ratti, R. A. (2014),Economic Policy Uncertainty and Firm-level Investment. Journal of Macroeconomics, 39, 42-53;

[14] Kapoor, M., Kelejian, H. H.\&Prucha, I. R. (2007),Panel Data Models with Spatially Correlated Error Components. Journal of econometrics, 140(1), 97-130;

[15] Kelejian, H. H.\&Prucha, I. R. (1999),A Generalized Moments Estimator for the Autoregressive Parameter in a Spatial Model. International economic review, 40(2), 509-533;

[16] Klößner, S.\&Sekkel, R. (2014),International Spillovers of Policy Uncertainty. Economics Letters, 124(3), 508-512;

[17] Krol, R. (2014),Economic Policy Uncertainty and Exchange Rate Volatility. International Finance, 17(2), 241-256;

[18] Liu, L.\&Zhang, T. (2015),Economic Policy Uncertainty and Stock Market Volatility. Finance Research Letters, 15, 99-105;

[19] Mumtaz, H.\&Theodoridis, K. (2015),The International Transmission of Volatility Shocks: An Empirical Analysis. Journal of the European Economic Association, 13(3), 512-533;

[20] Murtagh, F.\&Legendre, P. (2014),Ward's Hierarchical Agglomerative Clustering Method: Which Algorithms Implement Ward's Criterion?. Journal of classification, 31(3), 274-295

[21] Pástor, L.\&Veronesi, P. (2013),Political Uncertainty and Risk Premia. Journal of Financial Economics, 110(3), 520-545;

[22] Pesaran, M. H.\&Timmermann, A. (2005),Small Sample Properties of Forecasts from Autoregressive Models under Structural Breaks. Journal of Econometrics, 129(1-2), 183-217;

[23] Rapach, D. E., Strauss, J. K.\&Zhou, G. (2013),International Stock Return Predictability: What Is the Role of the United States?. The Journal of Finance, 68(4), 1633-1662. 